\title{
Use of Encapsulated Zinc Particles in a Eutectic Chloride Salt to Enhance Thermal Energy Storage Capacity for Concentrated Solar Power
}

\author{
Sreeram Cingarapu \\ Dileep Singh \\ Elena V. Timofeeva \\ Michael R. Moravek \\ Energy Systems Division \\ Argonne National Laboratory, Argonne, IL 60439, USA \\ *corresponding author e-mail: dsingh@anl.gov; phone: +1(630)-252-5009
}

\author{
Argonne National Laboratory \\ Argonne, IL 60439, USA \\ (E-mail: dsingh@anl.gov)
}

January 2015

The submitted manuscript has been created by Argonne National Laboratory, a U.S. Department of Energy laboratory managed by UChicago Argonne, LLC, under Contract No. DE-AC02-06CH11357 with the U.S. Department of Energy. The U.S. Government retains for itself, and others acting on its behalf, a paidup, nonexclusive, irrevocable worldwide license in said article to reproduce, prepare derivative works, distribute copies to the public, and perform publicly and display publicly, by or on behalf of the Government 


\section{Abstract}

Concentrated Solar Power (CSP) is considered as a viable large-scale renewable energy source to produce electricity. However, current costs to produce electricity from CSP are not cost competitive as compared to the traditional energy generation technologies based on fossil fuels and nuclear. It is envisioned that development of high efficiency and high heat capacity thermal storage fluids will increase system efficiency, reduce structural storage volume, and hence, contribute to reducing costs. Particularly, with respect to CSP, current high temperature energy storage fluids, such as molten salts, are relatively limited in terms of their thermal energy storage capacity and thermal conductivity. The current work explores possibility of boosting the thermal storage capacity of molten salts through latent heat of added phase change materials. We studied the advantage of adding coated $\mathrm{Zn}$ micron-sized particles to alkali chloride salt eutectic for enhanced thermal energy storage. Zinc particles $(0.6 \mu \mathrm{m}$ and $5 \mu \mathrm{m})$ obtained from commercial source were coated with an organo-phosphorus shell to improve chemical stability and to prevent individual particles from coalescing with one another during melt/freeze cycles. Thermal cycling tests (200 melt/freeze cycles) showed that coated $\mathrm{Zn}$ particles have good thermal stability and are chemically inert to alkali chloride salt eutectic in both $\mathrm{N}_{2}$ and in air atmospheres. Elemental mapping of the cross-sectional view of coated $\mathrm{Zn}$ particles from the composite after thermal cycles showed no signs of oxidation, agglomeration or other type of particle degradation. The measured enhancement in volumetric thermal storage capacity of the composite with just $\sim 10$ vol\% of coated $\mathrm{Zn}$ particles over the base chloride salt eutectic varies from $15 \%$ to $34 \%$ depending on cycling temperature range $\left(\Delta T=50^{\circ} \mathrm{C}-100^{\circ} \mathrm{C}\right)$.

\section{Keywords}

Encapsulation, phase change, thermal energy storage, eutectic salt, zinc, thermal conductivity. 


\section{Introduction}

The efficient use of renewable energy is of growing importance and developing highly effective energy storage solutions to balance intermittent nature of renewable energy sources is a paramount industry need. Concentrated solar power (CSP) is one of the promising renewable technologies that has the potential to offer large-scale grid-level power. However, the current costs to produce electricity from CSP are too high as compared to the other contemporary technologies that are based on fossil fuels, etc. To make CSP cost competitive, thermal energy storage (TES) systems with increased thermal energy storage capacity operating at high temperatures are required to improve the overall plant efficiency. Current high temperature storage systems provide only 6 - 7 hours of storage which is insufficient for continuous operation through the day/night cycles. It has been quoted that 13 hours storage can reduce CSP energy costs by $25 \%$ at annual capacity factor of 0.6-0.7 [1]. There are several types of CSP systems used for energy production through the steam turbines, namely the parabolic trough, power tower and parabolic dish $[2,3]$. Thermal energy is stored in the form of a sensible heat in either twotank storage system with separate hot and cold salt tanks, or in a single storage tank with a heat transfer fluid (HTF) [4]. The thermal storage capacity of those systems depends on the specific heat, thermal conductivity, heat transfer rates and the amount of HTF stored [4, 5]. The high temperature $\mathrm{HTFs}$ in the range from $250^{\circ} \mathrm{C}$ to $600^{\circ} \mathrm{C}$ that are considered for solar power systems are mainly inorganic alkali nitrate, alkali carbonate and alkali chloride salts (both pure substances and their eutectic mixtures). Mixing of nano- and micro-scale solid materials into HTFs are known to improve their thermal conductivity [5]. Additionally, a recent study has demonstrated that the specific heat of chloride-based HTFs can be improved by the addition of silica nanoparticles [6]. 
At the same time, storage of thermal energy in the form of latent heat of phase change materials $(\mathrm{PCM})$ has been demonstrated as an attractive strategy for low $\left(<150^{\circ} \mathrm{C}\right)$ and medium $\left(<250^{\circ} \mathrm{C}\right)$ temperature range applications. Addition of PCM to HTFs can contribute additional thermal energy storage capacity through the latent heat of solid/liquid or solid I/solid II transformations [4]. Typical PCMs for low temperature applications are organic compounds with very low thermal conductivities and heat transfer rates. A number of approaches to improve the thermal conductivity of PCMs have been investigated, including the addition of highly thermally conductive phases such as graphite [7, 8]. Further, encapsulation of organic PCMs into inorganic shells with higher thermal conductivity was found to increase the heat transfer rates [9, 10].

Several metal PCMs such as Li, In, Sn and eutectic alloys have also been evaluated for medium temperatures applications [11]. Tin nanoparticles $(\sim 100 \mathrm{~nm})$ encapsulated in silica shell were dispersed into organic HTF Therminol 66 and at 5 vol.\% nanoparticle loading, the thermal conductivity of composite HTF was improved by $13 \%$ and the volumetric thermal energy storage was also improved by $11 \%$ when the composite system was cycled between $100^{\circ} \mathrm{C}$ and $270^{\circ} \mathrm{C}$ [12].

Search for the higher efficiency HTF for high temperature CSP applications continues, and the use of encapsulated phase change materials (EPCM) chemically compatible with molten salts and with a phase transition occurring in a HTF's operating temperature range is of great interest. Addition of micro and nano-scale EPCM to a HTF allows for good dispersion stability, creates 
minimal obstructions to the flow, and offers dual property advantage such as increased specific heat due to the latent heat of PCM [7, 12] as well as enhanced thermal conductivity [12, 13]. In this study, zinc ( $\mathrm{Zn}$ ) was selected as a PCM because the melting temperature of $\mathrm{Zn}$ falls in the working range $\left(350-500^{\circ} \mathrm{C}\right)$ of a typical thermal energy storage eutectic salt formulations and has a high heat of fusion $(113 \mathrm{~J} / \mathrm{g})$ value [14]. First, commercial $\mathrm{Zn}$ particles of sizes $0.6 \mu \mathrm{m}$ and 5.0 $\mu \mathrm{m}$ were coated with organo-phosphorous compound (trioctyl phosphine oxide, TOPO) to protect from segregation of particles when melted as well as to provide protective layers around the particles to prevent metal oxidation. Second, the thermal stability of TOPO-coated $\mathrm{Zn}$ particles was investigated by thermal cycling (200 heat/cool cycles) in nitrogen and in air atmosphere. Next, coated $\mathrm{Zn}$ particles were dispersed in chloride salt eutectic at various volumetric loadings and the thermal analysis was carried out using differential scanning calorimetry. The effects of concentration and sizes of coated $\mathrm{Zn}$ particles $(0.6 \mu \mathrm{m}$ vs. $5.0 \mu \mathrm{m})$ on the total heat adsorption by the composite were also investigated. Increase in thermal conductivity of the molten salt due to addition on $\mathrm{Zn}$ particles was estimated from the Maxwell's effective medium theory. The effective specific heat, $C_{p(\text { composite })}$, and density, $\rho_{\text {(composite })}$ of the composite at various temperatures were calculated using the rule of mixtures.

\section{Materials and Methods}

Zinc particles of two different sizes $(0.6 \mu \mathrm{m}$ and $5 \mu \mathrm{m})$ were obtained from Sigma Aldrich (phase purity 99\%). The containers of the $\mathrm{Zn}$ particles were opened in $\mathrm{N}_{2}$ atmosphere glove box to minimize oxidation by air, and a small amount of each sample was used to characterize the asreceived particles. The powder X-ray diffraction (PXRD) patterns of as-received Zn particles 
were recorded by a Bruker D8 X-ray diffractometer with $\mathrm{Cu} \mathrm{K}_{\alpha}$ radiation from $2 \theta$ of $20^{\circ}$ to $80^{\circ}$ at increments of $1 \% \mathrm{~min}$.

Morphology and particle sizes of as-received $\mathrm{Zn}$ particles were studied with scanning electron microscopy (SEM, Hitachi Model -4700 -II, Tokyo, Japan) and transmission electron microscopy (TEM, Philips CM 300). The samples for microscopy studies were prepared by placing a drop of dilute suspension of particles in ethanol onto a silicon wafer (for SEM) or on to a porous carbon coated copper grid (for TEM) and allowed to dry before examination. Focused ion beam (FIB) was used to investigate the cross sections of particles as well. Additionally, ethanol dispersion of particles was used to estimate the average particle sizes with the dynamic light scattering (DLS) technique (90plus/BIMAS particle analyzer, Brookhaven Instrument Corp, NY).

Trioctyl phosphine oxide (TOPO) was obtained from Sigma Aldrich (reagent plus 99\%). TOPO is widely used in industry as a ligand for the extraction of various metals [15-17]. It has been used as a solvent and capping molecule in the synthesis of a wide range of nanoparticles $[18,19]$. Chemical structure of TOPO (three octyl carbon chains resulting in local trigonal symmetry, and a large permanent dipole moment from the $\mathrm{P}=\mathrm{O}$ bond) results in ability to form self-assembled monolayers and at higher concentration it forms multilayered structures [20] and protects nanomaterials from oxidation $[21,22]$. Coating of zinc particles with TOPO was carried out by the mixing of an appropriate amount of TOPO with Zn-particles (1:10 by weight), followed by slow melting of $\mathrm{TOPO}\left(\right.$ at $55^{\circ} \mathrm{C}$ ) onto the $\mathrm{Zn}$ surface. All sample preparation procedures were carried out inside the $\mathrm{N}_{2}$ atmosphere glove box. 
153 Alkali chloride salt eutectic was obtained from Texas A\&M University. Composition and preparation of the eutectic salt are described elsewhere [6]. Nominal melting of the salt occurs at $400^{\circ} \mathrm{C}$ and freezing around $380^{\circ} \mathrm{C}$. The composites of eutectic salt with TOPO coated zinc particles (Zn-TOPO) were prepared by placing the appropriate amounts of coated particles and salt in a crucible and heating the crucible on a hot plate until complete salt melting was achieved. Residual TOPO was washed off with ethanol.

A differential scanning calorimeter (DSC) Q-20 (TA Instruments) was used to measure the specific heat, heat of fusion, melting and crystallization temperatures of as-received zinc samples, encapsulated $\mathrm{Zn}$-TOPO particles and the eutectic salt composite with various volume loadings of Zn-TOPO particles. The nitrogen and air were used as purge gases in those tests. Before the experiments the instrument was calibrated against the melting point and enthalpy of melting of indium and tin standards. Specific heat was measured using ASTM-E1269 standard method. Sapphire was used for calibration and the measured values were with $2.5 \%$ of the standard values over the temperature range used in this study.

\section{Results and Discussion}

Characterization of $Z n$ particles

The XRD patterns (Figure 1) of both as-received Zn powders $(0.6 \mu \mathrm{m}$ and $5.0 \mu \mathrm{m})$ exhibit a prominent X-ray diffraction peaks at scattering angles $2(\theta)$ of $36.2,38.9,43.2,54.3,70.1$ and 70.6 characteristic for metallic zinc and correspond to (002), (100), (101), (102), and (103) lattice planes of hexagonal phase of zinc, respectively. The XRD peaks at $\sim 70$ degrees are higher 
compared to the database peak ratio, which often happens due to morphology of the particles and their preferential alignment on the sample stage. [23]. No peaks of oxide impurities were observed in XRD pattern confirming that the obtained Zn particles are phase pure metal [24].

SEM imaging showed spherical shape of both types of as-received Zn powders. Some variation in particle size is observed ranging from $0.2-0.8 \mu \mathrm{m}$ for powder with nominal size of $0.6 \mu \mathrm{m}$ and 1-10 $\mu \mathrm{m}$ for powder with nominal size of $5 \mu \mathrm{m}$ (Figure 2a and $2 \mathrm{~b}$ ). Average particle sizes were also measured with DLS showing number based average sizes as $0.64 \pm 0.1 \mu \mathrm{m}$ and $5.3 \pm 0.9 \mu \mathrm{m}$ which is in a good agreement with manufacturer specifications.

\section{Study of as-received Zn particles}

The melting and freezing temperatures and the latent heat of fusion of as-received particles were measured calorimetrically. Phase change was observed at $418.1{ }^{\circ} \mathrm{C}$ and at $414.5{ }^{\circ} \mathrm{C}$ for melting and freezing, respectively similar for both particle sizes (Figure 3), which is slightly lower than the reported freezing point value for bulk $\mathrm{Zn}$ metal $\left(419.6^{\circ} \mathrm{C}\right)$ [14]. The values of the latent heat of fusion from the first measurement in air atmosphere shows $97.7 \mathrm{~J} / \mathrm{g}$ and $103.0 \mathrm{~J} / \mathrm{g}$ for $0.6 \mu \mathrm{m}$ and $5.0 \mu \mathrm{m}$ particles, which are $13 \%$ and $9 \%$ lower than the value reported for bulk $\mathrm{Zn}$ metal $(112 \mathrm{~J} / \mathrm{g})$ [14]. Besides lower values on the first thermal cycle, the experimental values of latent heat of fusion further decreased from $97.7 \mathrm{~J} / \mathrm{g}$ to $44.4 \mathrm{~J} / \mathrm{g}$ for $0.6 \mu \mathrm{m}$ particles (Figure $4 \mathrm{a}$ ) and from $103.0 \mathrm{~J} / \mathrm{g}$ to $86.0 \mathrm{~J} / \mathrm{g}$ for $5 \mu \mathrm{m}$ particles (Figure $4 \mathrm{~b}$ ) after just 10 thermal cycles in air. These numbers translate into $55 \%$ and $17 \%$ capacity loss for $0.6 \mu \mathrm{m}$ and $5 \mu \mathrm{m}$ particles respectively, 
which clearly indicates chemical instability of $\mathrm{Zn}$ particles in air resulting in metal oxidation. Also, it can be concluded that smaller particles with higher surface area are more reactive. Therefore, the difference in experimentally measured latent heat of fusion for $0.6 \mu \mathrm{m}$ particles, $5.0 \mu \mathrm{m}$ particles, and the literature values for bulk $\mathrm{Zn}$ can be explained by the presence and continued formation of surface oxides when $\mathrm{Zn}$ particles are exposed to oxygen in the air. The rate of surface oxide formation is a function of the particle surface area and the temperature.

\section{Study of TOPO coated Zn particles}

To improve chemical and thermal stability of $\mathrm{Zn}$ particles, the powders were coated with organophosphate compound TOPO. Coated Zn particles were calorimetrically tested under air and $\mathrm{N}_{2}$ atmosphere and results are also presented on Figure $4 \mathrm{a}(0.6 \mu \mathrm{m}$ particles $)$ and Figure $4 \mathrm{~b}$ (5 $\mu \mathrm{m}$ particles). In both cases coated particles demonstrated very stable values of the heat of fusion in both $\mathrm{N}_{2}$ and in air purge. These results confirm that coating of the particles with TOPO prevents $\mathrm{Zn}$ surface oxidation and provides stable phase change performance over 200 thermal cycles. The average value of the latent heat of fusion for coated $0.6 \mu \mathrm{m} \mathrm{Zn}$ particles was $70 \mathrm{~J} / \mathrm{g}$ in $\mathrm{N}_{2}$ and $82 \mathrm{~J} / \mathrm{g}$ in air atmosphere (Figure $4 \mathrm{a}$ ). For coated $5.0 \mu \mathrm{m}$ size $\mathrm{Zn}$ particles, the average values were $97 \mathrm{~J} / \mathrm{g}$ in $\mathrm{N}_{2}$ and $103 \mathrm{~J} / \mathrm{g}$ in air atmosphere (Figure 4b). It should be noted that the specific heat of fusion for TOPO coated $0.6 \mu \mathrm{m} \mathrm{Zn}$ particles is $\sim 15 \%$ lower than the initial value for as-received $0.6 \mu \mathrm{m}$ particles (Figure 4a). In case of $5 \mu \mathrm{m} \mathrm{Zn}$ particles the heat of fusion values are the same for TOPO coated and as-received (Figure 4b). This result is most likely related to $\sim 9$ times higher surface area of $0.6 \mu \mathrm{m}$ vs. $5.0 \mu \mathrm{m}$ particles, and therefore, higher mass fraction of TOPO attached to the surface and surface oxides. Interestingly, for both particle sizes 
thermal cycling in $\mathrm{N}_{2}$ atmosphere resulted in $8-15 \%$ lower latent heat values when compared to the thermal cycling in air (Figure 4). At the present time, the reason for this observation is not clear, but, could be related to interaction between TOPO and carrier gases at elevated temperatures.

\section{Study of eutectic salts composites with Zn-TOPO particles}

To further demonstrate chemical and thermal stability of the coated $\mathrm{Zn}$ particles, they were added to the alkali eutectic chloride salt at different volume loadings and 200 repeated thermal cycles $\left(300-500^{\circ} \mathrm{C}\right)$ were carried out in both $\mathrm{N}_{2}$ and in air atmosphere (for a consistent data representation only 20 (Figure $4 \mathrm{a}$ ) and 100 cycles (Figure 4b) are shown). Since the composite (salt mixed with Zn-TOPO particles) was synthesized in a glove box the loadings were determined by the weight additions and vary from sample to sample between 8 and 22\% by volume.

For composite with $0.6 \mu \mathrm{m}$ particles an average heat of fusion value of $\sim 20 \mathrm{~J} / \mathrm{g}$ due to $\mathrm{Zn}$ melting were measured in both $\mathrm{N}_{2}$ for 11.5 vol. $\%$ and in air atmosphere for 13.0 vol.\% of $\mathrm{Zn}$-TOPO composites respectively (Figure 4a). For composites with $5.0 \mu \mathrm{m}$ coated particles average latent heat of fusion values were $41 \mathrm{~J} / \mathrm{g}$ at $21 \mathrm{vol} . \%$ in $\mathrm{N}_{2}$ and $27 \mathrm{~J} / \mathrm{g}$ at $15 \mathrm{vol} \%$ in air. All reported values were stable over 200 thermal cycles.

\section{Study of morphological changes due to thermal cycling and exposure}


The TEM images of TOPO coated $0.6 \mu \mathrm{m}$ Zn particles before (Figure 5a) and after (Figure 5b) 20 thermal cycles (salts were washed off with ethanol) show no obvious changes in the particle morphology. For comparison SEM and TEM and corresponding elemental mapping images of FIB sliced cross-section shown for as received $\mathrm{Zn}$ particles after one hour exposure to air at $400^{\circ} \mathrm{C}$ are presented in Figure 6 (a-c). The particle degradation is observed on those images as compared to relatively pristine surface of zinc as shown in Figure $2 b$.

The continuous oxidation of metallic $\mathrm{Zn}$ into $\mathrm{ZnO}$ explains $55 \%$ decrease in specific heat of fusion over first 10 cycles for $\mathrm{Zn}$ particles that were not coated. Other studies also reported considerable change in the surface texture upon thermal cycles of $\mathrm{Zn}$ in air atmosphere, which also were prescribed to the formation of surface oxides and densification during thermal cycles $[14,21]$.

SEM and EDAX images of eutectic salt with $5.0 \mu \mathrm{m}$ Zn-TOPO particles after 200 cycles of thermal cycling in $\mathrm{N}_{2}$ and in air atmosphere are presented on Figure 7 and Figure 8, respectively. The EDAX elemental mappings of the composite cross-section (Figure 7b and 8c) show different elements (O-yellow, Cl- blue, Ca- purple, Ba-magenta and $\mathrm{Zn}$-red) that are present in the composite. Calcium, barium and chlorine are from the eutectic salt, and the trace amount of oxygen found next to the $\mathrm{Cl}$ and $\mathrm{Ba}$ most likely is from the oxidation of Ba salt. The SEM of a cross-section of the Zn-TOPO particle cycled 200 times in $\mathrm{N}_{2}$ atmosphere (Figure $7 \mathrm{a}$ ) shows no change in density of particles, no significant signs of oxidation, shell formation or particle aggregation. Elemental map (Figure 7b) shows no oxygen inside the particle, just minor amount on the surface of which could be due to TOPO coating. 
263 Maps of the composite with $\mathrm{Zn}$-TOPO particles cycled in air have higher concentration of oxygen, but it can be seen, that there is significant fraction of $\mathrm{Zn}$ particles that don't have any oxygen on the surface. On the other hand SEM of FIB milled cross-section (Figure 8b) shows obvious formation of crust around the metallic $\mathrm{Zn}$ particle. From the elemental map however it appears to be formed by wetting of the particle with the eutectic salt and possibly reaction between the salt, air and the particle surface. Also, it should be noted that oxygen is present mostly in the area where $\mathrm{Ba}$ and $\mathrm{Cl}$ is present, so formation of $\mathrm{BaO}$ can be suggested.

From the TEM image (Figure 8d) TOPO appears to form as a thick protective layer around the Zn-particle. It is known that due to its chemical structure (three octyl carbon chains resulting in local trigonal symmetry, and a large permanent dipole moment from the $\mathrm{P}=\mathrm{O}$ bond) TOPO can also form self-assembled monolayers. At higher concentration, TOPO can form multilayered structures [20] and protects nanomaterials from oxidation [21, 22]. Elemental mapping on the FIB'ed particles shows no evidence of surface oxygen before thermal cycling (Figure 8e) and also after thermal cycling in salt (Figure 8c). The SEM and elemental mappings confirm that the TOPO coated $\mathrm{Zn}$ particles are thermally and chemically stable, which is further attested by the stable values for the heat of fusion through hundreds of melt/freeze cycles.

\section{Thermal energy storage in composites Zn-TOPO particles}

Latent heat of fusion from melting of $\mathrm{Zn}$ nanoparticles adds to the total energy storage capacity of the eutectic salt composite Obtained experimental values were compared against the calculated values for the volumetric heat capacity of the composite with the $\mathrm{Zn}$-TOPO particles. 
The effective specific heat, $C_{p(\text { composite })}$, and density, $\rho_{\text {(composite })}$ of the composite at various temperatures were calculated using the rule of mixtures (Eq. $1 \& 2$ ), and total volumetric heat capacity was calculated using equation 3 :

Effective specific heat:

$$
C_{p(\text { composite })}=\frac{\left(1-\phi_{Z n}\right) C_{p(\text { salt })} \rho_{(\text {salt })}+\phi_{(Z n)} C_{p(Z n)} \rho_{(Z n)}}{\left(1-\phi_{(Z n)}\right) \rho_{(s a l t)}+\phi_{(Z n)} \rho_{(Z n)}}
$$

Effective density of composite:

$$
\rho_{(\text {composite })}=\rho_{(Z n)} \phi_{(Z n)}+\left(1-\phi_{(Z n)}\right) \rho_{(\text {salt })}
$$

Volumetric Thermal Energy Storage (TES):

$$
\Delta \mathrm{Q}_{(\text {composite })}=\rho_{(\text {composite })} C_{p(\text { composite })} \Delta T+\rho_{(Z n)} \phi_{(Z n)} \Delta \mathrm{H}_{f(Z n)}
$$

where, $C_{p}$ (composite) is specific heat capacity of mixture (Zn-TOPO particles mixed in eutectic salt), $C_{p(\text { salt })}$ is specific heat of base eutectic salt, $C_{p(Z n)}$ is the specific heat of Zn-TOPO particles, $\phi_{(Z n)}$ is volume fraction of Zn-TOPO particles, $\rho_{(\text {salt })}$ is density of base eutectic salt, $\rho_{(\mathrm{Zn})}$ is density of Zn-TOPO particles, $\rho_{(\text {composite })}$ is the effective density of mixture, $\Delta T$ is the difference in temperature measured from $T_{1}$ to $T_{2}\left({ }^{\circ} \mathrm{C}\right)$, and $\Delta \mathrm{H}_{f(Z n)}$ is the heat of fusion of Zn-TOPO particles.

The experimental total heat absorption by a composite of alkali chloride salt eutectic with $0.6 \mu \mathrm{m}$ TOPO coated $\mathrm{Zn}$ particles was determined by thermal cycling between $300^{\circ} \mathrm{C}$ and $500^{\circ} \mathrm{C}$. Figure 9 shows a typical DSC graph for the composite system.. During heating, the endothermic peaks at $395.0^{\circ} \mathrm{C}$ and $417.1^{\circ} \mathrm{C}$ correspond to the melting of the alkali chloride eutectic salt and encapsulated Zn particles, respectively. The two exothermic peaks appear during 
cooling of the cycle at $411.5^{\circ} \mathrm{C}$ and $385.0^{\circ} \mathrm{C}$ correspond to the freezing of $\mathrm{Zn}$-TOPO particles and eutectic salt, respectively.

The measured specific heat of alkali chloride salt eutectic in $\mathrm{N}_{2}$ atmosphere at $350^{\circ} \mathrm{C}$ is 1.07 $\mathrm{J} / \mathrm{g}^{*} \mathrm{~K}$ and at $500^{\circ} \mathrm{C}$ it is $1.17 \mathrm{~J} / \mathrm{g}^{*} \mathrm{~K}$. The specific heat of $\mathrm{Zn}$-TOPO particles was assumed to be constant in the given range of temperatures $\left(300^{\circ} \mathrm{C}-500^{\circ} \mathrm{C}\right)$. Values of measured and calculated specific heat for 8.5 vol.\% of $\mathrm{Zn}$ particles loading are presented in Table 1. One can see that after 20 cycles the measured specific heat values are $\sim 16-20 \%$ smaller than calculated values. The added heat of fusion from melting of $\mathrm{Zn}$ particles in the composite was measured to be $17.6 \mathrm{~J} / \mathrm{g}$ which is also $20 \%$ less than the calculated value of $22.1 \mathrm{~J} / \mathrm{g}$ (from Eq. 3 ).

As seen from Eq. 3 the resulting contribution of phase change material to the total TES will depend on the temperature range in which the material is being cycled $(\Delta T)$. Figure 10 shows average increases in volumetric TES capacity for 10 vol.\% loading of $\mathrm{Zn}$ particles in alkali chloride salt eutectic as a function of the cycling temperature range $(\Delta T)$. The line curve represents the calculated values (per Eq. 3) based on the measured specific heat of alkali chloride salt eutectic, density of alkali chloride salt eutectic and Zn-TOPO particles, and the experimental value for the heat of fusion of $\mathrm{Zn}$-TOPO particles. The volumetric TES enhancement is strongly dependent on the cycling temperature range for the composite HTF. It should be noted that the smaller the $(\Delta T)$, the higher is the effect of adding EPCM particles. As $\Delta T$ increases, the specific heat of the composite fluid (sensible heat) has a higher contribution than the heat absorption by melting of EPCM particles. The predicted increase in volumetric TES, $\Delta Q_{\text {(composite) }}$ of alkali chloride salt eutectic with 10 vol.\% of coated $\mathrm{Zn}$ particles is $\sim 45 \%$ for 
the temperature range $(\Delta T)$ of $50^{\circ} \mathrm{C}$ and the measured value is $34 \pm 7 \%$ for $\Delta T$ between $400^{\circ} \mathrm{C}$ and $450^{\circ} \mathrm{C}$. If the cycling temperature range is further expanded to $400^{\circ} \mathrm{C}-500^{\circ} \mathrm{C}$ the benefit of adding Zn-TOPO particles is further decreased to $15 \pm 3 \%$ (calculated value at $\Delta T$ of $100^{\circ} \mathrm{C}$ is $\left.23 \%\right)$.

Besides enhancing the volumetric TES of the eutectic salt, addition of Zn-TOPO particles should contribute to enhancing the thermal conductivity of the composite. Base thermal conductivity of eutectic salts is very low $1-2 \mathrm{~W} / \mathrm{mK}$, as compared to $116 \mathrm{~W} / \mathrm{mK}$ for metallic Zn. According to Maxell's effective medium theory [25], at 10 vol.\% of Zn particles, thermal conductivity of molten salt should also increase $\sim 30 \%$. Furthermore increase in concentration of EPCM Zn particles will result in even higher benefits to both TES and thermal conductivity, however addition of large volumetric fractions of EPCM particles to molten salt may modify rheological properties of the fluid, increasing viscosity and pumping power requirements.

It is interesting to note that in most experiments we have observed consistent $\sim 20 \%$ difference between the experimental and calculated values. This could be due to an experimental error and/or a variation in the actual composition due to the limited accuracy in preparation of small batches. Also using literature values for $C_{p(Z n)}$ and $\rho_{(Z n)}$ causes overestimation in calculated values for the composite, especially for the case of $0.6 \mu \mathrm{m} \mathrm{Zn}$ particles, since coating with TOPO reduces the effective specific heat of $\mathrm{Zn}$ particles by $\sim 18 \%$ (Figure 4 ). For this and other economic reasons use of larger, e.g. $5 \mu \mathrm{m}$ Zn-TOPO particles as EPC materials, may be 
preferential. Nonetheless, our experiments demonstrate that the TES of molten salts can be significantly improved by addition of EPC particles.

In summary, chemical and thermal stability of commercial $\mathrm{Zn}$ particles (0.6 $\square \mathrm{m}$ and $5 \square \mathrm{m}$ ) was achieved by application of organo-phosphorus coating (TOPO). Further, coated Zn particles (ZnTOPO, EPCM-particles) were found to be chemically inert to alkali chloride salt eutectic, and thermally stable in multiple heat/cooling cycles in both $\mathrm{N}_{2}$ and in air atmosphere, as confirmed by the elemental mapping (EDAX) of the composite after melt/freeze cycles. Addition of EPCM Zn particles to the eutectic salt increases thermal energy storage capacity over the base salt due to $\mathrm{Zn}$ latent heat of fusion for the value proportional to the cycling temperature range and volumetric concentration of Zn particles. EPCM Zn particles offer dual benefits by increasing the TES and potentially increase in thermal conductivity of eutectic salt

\section{Conclusions}

The current work focuses on improving the thermal energy storage for concentrated solar power generation. Addition of encapsulated phase change $\mathrm{Zn}$ particles to alkali chloride eutectic salt can significantly improve thermal energy storage capacity and thermal conductivity of this heat transfer fluid. $\mathrm{Zn}$ particles tend to oxidize on air, rapidly converting to $\mathrm{ZnO}$ and loosing ability to phase change. In this study we have demonstrated that coating of as-received $\mathrm{Zn}$ particles with appropriate amount of organo-phosphorous compound establishes a barrier to $\mathrm{Zn}$ oxidation and provides thermal stability to the particle agglomeration when melted. Encapsulated $\mathrm{Zn}$ particles have demonstrated chemical inertness towards the high temperature alkali chloride salt eutectics 
379 in more than 200 melt/freeze cycles. Improved thermal energy storage and thermal conductivity

380 of high temperature heat transfer fluids due to addition of coated $\mathrm{Zn}$ particles will increase the 381 system efficiency by up to $45 \%$ at only 10 vol. $\%$ of particles, which in turn will reduce structural 382 storage volumes, and will contribute in bringing costs of solar power generation in line with 383 other conventional power generation technologies.

\section{Acknowledgments}

We appreciate help from Dr. Jon Hiller and Dr. Rachel E. Koritala in conducting FIB-SEM and TEM. This work was supported by US Department of Energy's EERE Solar Energy Technology Program - ARRA funding. Fruitful discussions with DOE project monitors Joe Stekli and Levi Irwin are much appreciated. The electron microscopy was accomplished at the Electron Microscopy Center for Materials Research at Argonne National Laboratory, a U.S. Department of Energy Office of Science Laboratory operated under Contract No. DE-AC02-06CH11357 by 


\section{References:}

1. Concentrating Solar Power: its potential contribution for a sustainable energy future, European Academies Science Advisory Council Policy Report 16, November 2011.

2. Herrmann, U., and D.W. Kearney. 2002. Survey of thermal energy storage for parabolic trough power plants. Journal of Solar Energy Engineering-Transactions of the ASME 124:145-152.

3. Emerging Energy Research (2010), Global Concentrated Solar Power Markets and Strategies: 2010- 2025, IHS, Cambridge, MA.

4. Yang M, Yang X, Yang X, Ding J. Heat transfer enhancement and performance of the molten salt receiver of a solar power tower. Applied Energy, 2010; 87: 2808e11.

5. Choi, S. U. S. "Enhancing thermal conductivity of fluids with nanoparticles." ASMEPublications-Fed 231 (1995): 99-106. .

6. Shin, D., and D. Banerjee. 2011. Enhancement of specific heat capacity of hightemperature silica-nanofluids synthesized in alkali chloride salt eutectics for solar thermalenergy storage applications. International Journal of Heat and Mass Transfer 54:10641070.

7. Steinmann, W.-D., M. Eck, and D. Laing. 2005. Solar thermal parabolic trough power plants with integrated storage capacity. International Journal of Energy Technology and Policy 3:123-336.

8. Zalba, B., Marin, J.M., Cabeza, L.F., Mehling, H. (2003). Review on thermal energy storage with phase change, heat transfer analysis and applications. Applied Thermal Engineering, 23, 251-283.

9. Chen, Johnson C.H., et. al., Encapsulated Phase Change Thermal Energy Storage Materials, United States Patent (1985).

10. Colvin, D.P., "Enhanced Thermal Management Using Encapsulated Phase Change Materials An Overview", Advances in Heat and Mass Transfer in Biotechnology, HTDVol. 363, BED-Vol. 44, pp199-206 (1999).

11. Hoshi, A., Mills, D.R., Bittar, A., Saitoh, T.S. (2005). Screening of high melting point phase change materials (PCM) in solar concentrating technology based on CLFR. Solar Energy, 79 (3), 332-339. 
12. S. Cingarapu, D. Singh, E.V. Timofeeva, M.R. Moravek, Nanofluids with Encapsulated Tin Nanoparticles for Advanced Heat Transfer and Thermal Energy Storage, Int. J. Energy Research, 38 (1) 51-59 2014,.

13. Timofeeva, E.V., M.R. Moravek, and D. Singh, Improving the heat transfer efficiency of synthetic oil with silica nanoparticles. Journal of Colloid and Interface Science, 2011. 364(1): p. 71-79.

14. Gunawan, L., and G.P. Johari. 2008. Specific Heat, Melting, Crystallization, and Oxidation of Zinc Nanoparticles and Their Transmission Electron Microscopy Studies. Journal of Physical Chemistry C 112:20159-20166

15. Watson, E.K., and W.A. Rickelton. 1992. A review of the industrial and recent potential applications of trioctylphosphine oxide Solvent Extraction and Ion Exchange 10:879-889

16. Chen, Q.Y., L.A. Li, L. Bai, H.P. Hu, J.A. Li, Q.W. Liang, and J.H. Ling. 2011. Synergistic extraction of zinc from ammoniacal ammonia sulfate solution by a mixture of a sterically hindered beta-diketone and tri-n-octylphosphine oxide (TOPO). Hydrometallurgy 105:201-206.

17. Matsui, M., T. Aoki, H. Enomoto, and T. Shigematsu. 1975. Non-aqueous liquid-liquidextraction- Extraction of zinc from ethylene-glycol solution of chloride by trioctylphosphine oxide. Analytical Letters 8:247-255

18. Collier, C.P., T. Vossmeyer, and J.R. Heath. 1998. Nanocrystal superlattices Annual Review of Physical Chemistry 49:371-404

19. Murray, C.B., D.J. Norris, and M.G. Bawendi. 1993. Synthesis and characterization of nearly monodisperse $\mathrm{CdE}(\mathrm{E}=$ sulfur, selenium, tellurium) semiconductor nanocrystallites. Journal of the American Chemical Society 115:8706-8715.

20. Jiang, J., T.D. Krauss, and L.E. Brus. 2000. Electrostatic force microscopy characterization of trioctylphosphine oxide self-assembled monolayers on graphite. Journal of Physical Chemistry B 104:11936-11941

21. Bi, Y., Y.B. Yuan, C.L. Exstrom, S.A. Darveau, and J.S. Huang. 2011. Air Stable, Photosensitive, Phase Pure Iron Pyrite Nanocrystal Thin Films for Photovoltaic Application. Nano Letters 11:4953-4957

22. Schuh, C.A., and D.C. Dunand. 2002. Enhanced densification of zinc powders through thermal cycling. Acta Materialia 50:1349-1358. 
23. Powder diffraction database PDF 03-065-5973.

457

24. Powder diffraction database PDF 03-065-3358.

458 25. Maxwell, J. C., A Treatise on Electricity and Magnetism, II, ed., Claredon, Oxford, 1881. 459 
Table 1: Measured and calculated specific heat of composite with $\sim 8.5 \mathrm{vol} \%$ of $\mathrm{Zn}$ particle loading.

461

462

\begin{tabular}{|l|l|l|l|}
\hline Temperature, ${ }^{\circ} \mathrm{C}$ & $\begin{array}{l}\text { Measured } \\
\text { specific } \\
\text { heat at } 1^{\text {st }} \\
\text { cycle, } \\
\mathrm{J} / \mathrm{g} * \mathrm{~K}\end{array}$ & $\begin{array}{l}\text { Measured } \\
\text { specific } \\
20^{\text {th }} \text { cycle, } \\
\mathrm{J} / \mathrm{g} * \mathrm{~K}\end{array}$ & $\begin{array}{l}\text { Calculated } \\
\text { specific heat } \\
\text { values, } \\
\mathrm{J} / \mathrm{g} * \mathrm{~K}\end{array}$ \\
\hline 450 & 1.072 & 0.921 & 1.073 \\
\hline 475 & 0.889 & 0.874 & 1.047 \\
\hline 495 & 0.907 & 0.854 & 1.024 \\
\hline
\end{tabular}

463

464

465 


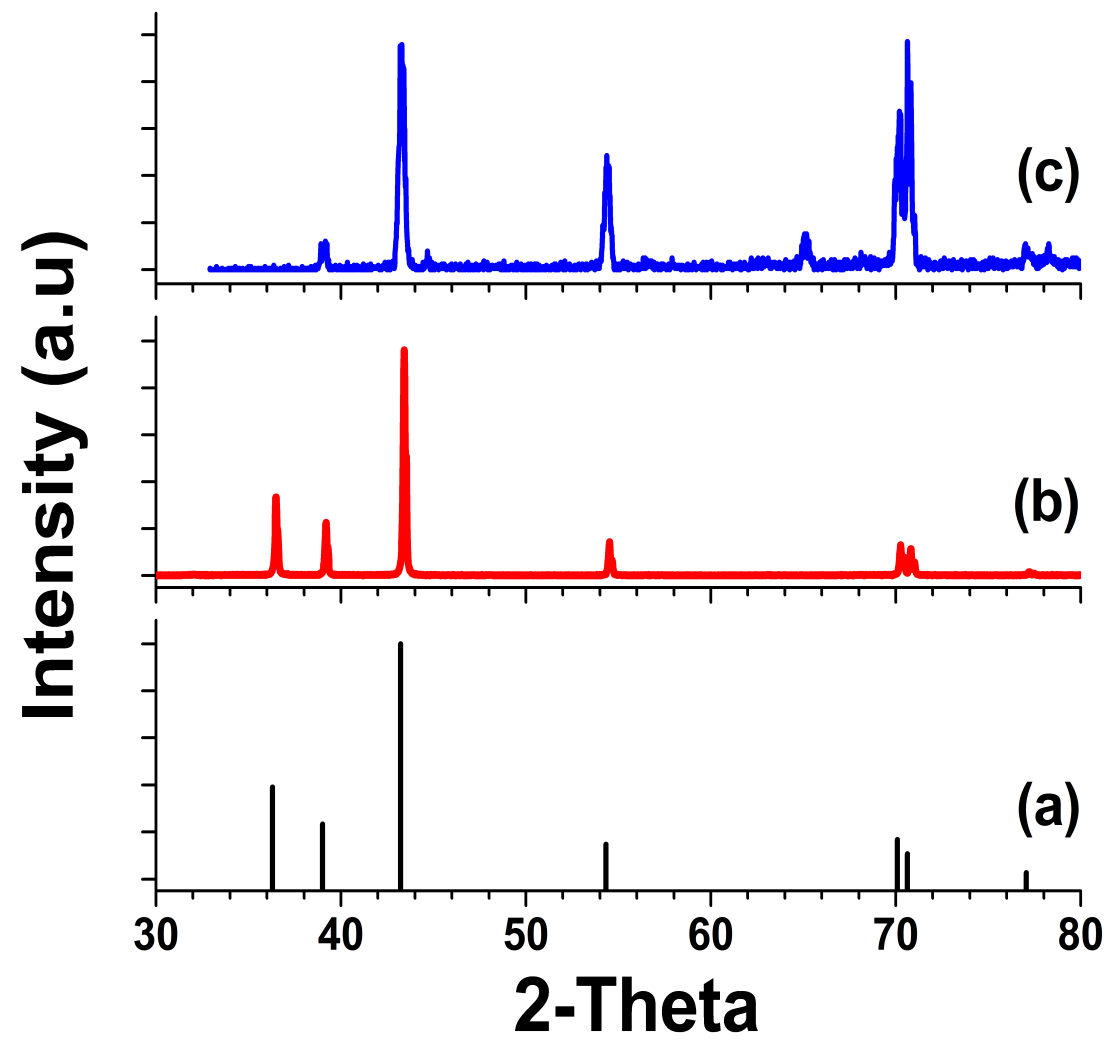

467 Figure 1: X-ray diffraction patterns of (a) reference PDF 03-065-3358 [24] and as-received zinc 468 particles (b) $0.6 \mu \mathrm{m}$ and (c) $5.0 \mu \mathrm{m}$ showing all the characteristic zinc metal peaks. 


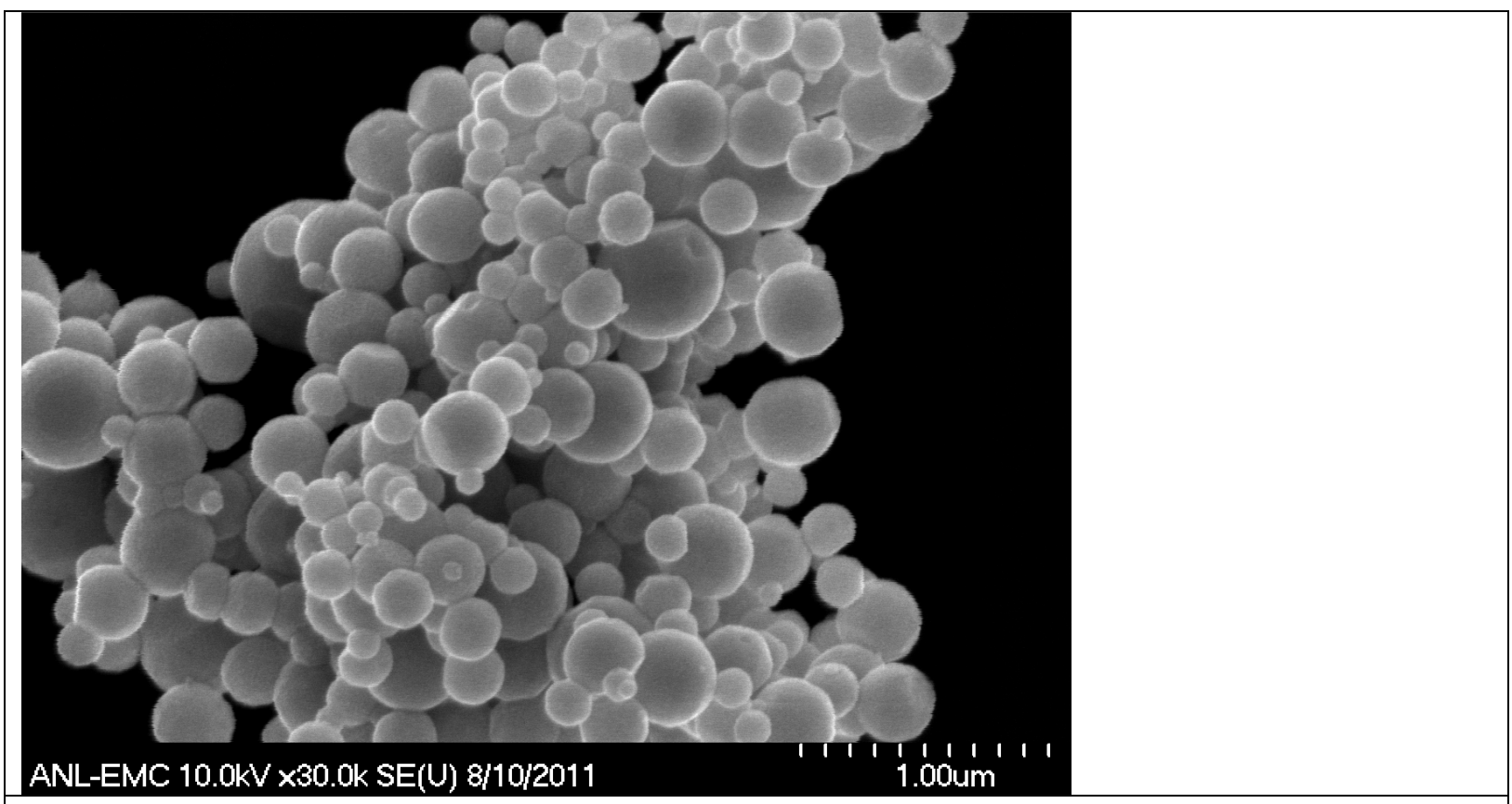

(a)

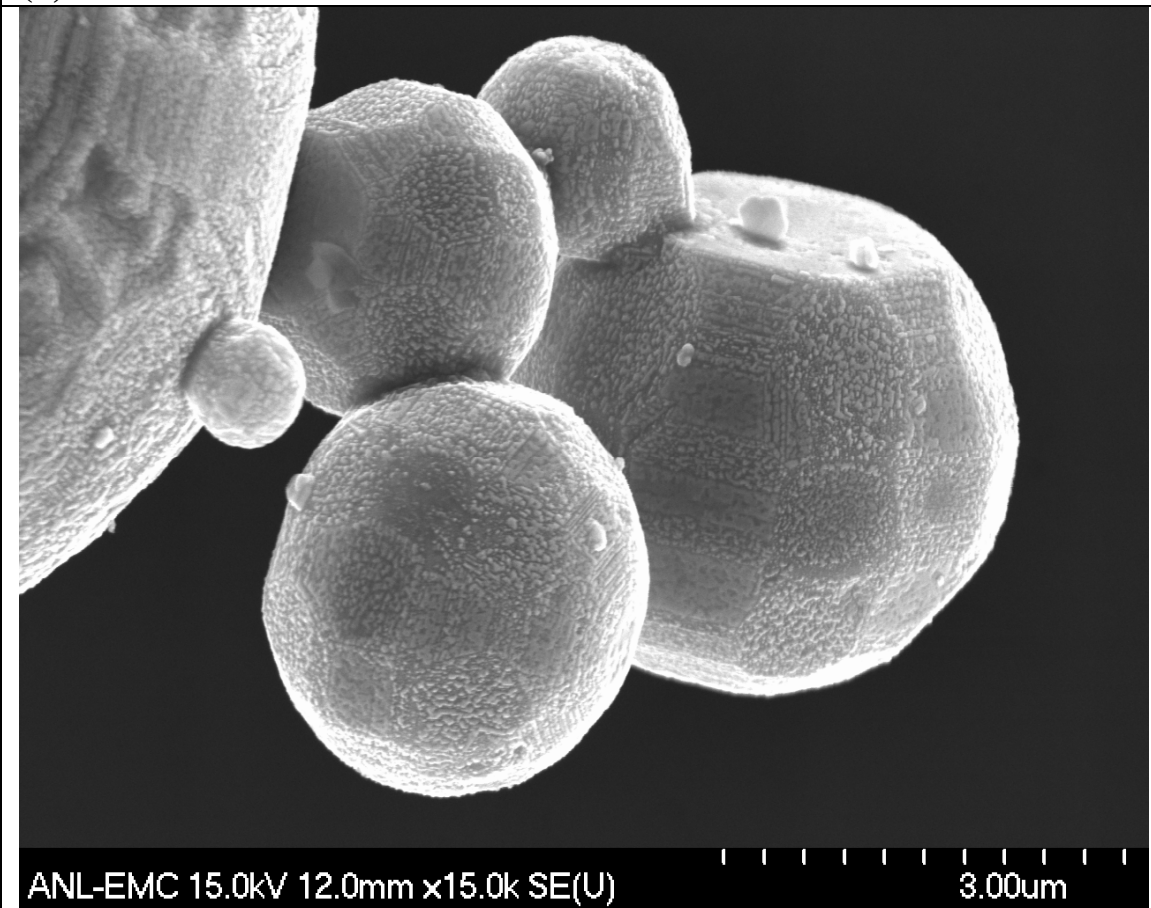

(b)

Figure 2: Scanning electron microscopy images of as-received $\mathrm{Zn}$ powders with nominal sizes of (a) $\sim 0.6 \mu \mathrm{m}$ and (b) $\sim 5.0 \mu \mathrm{m}$. 


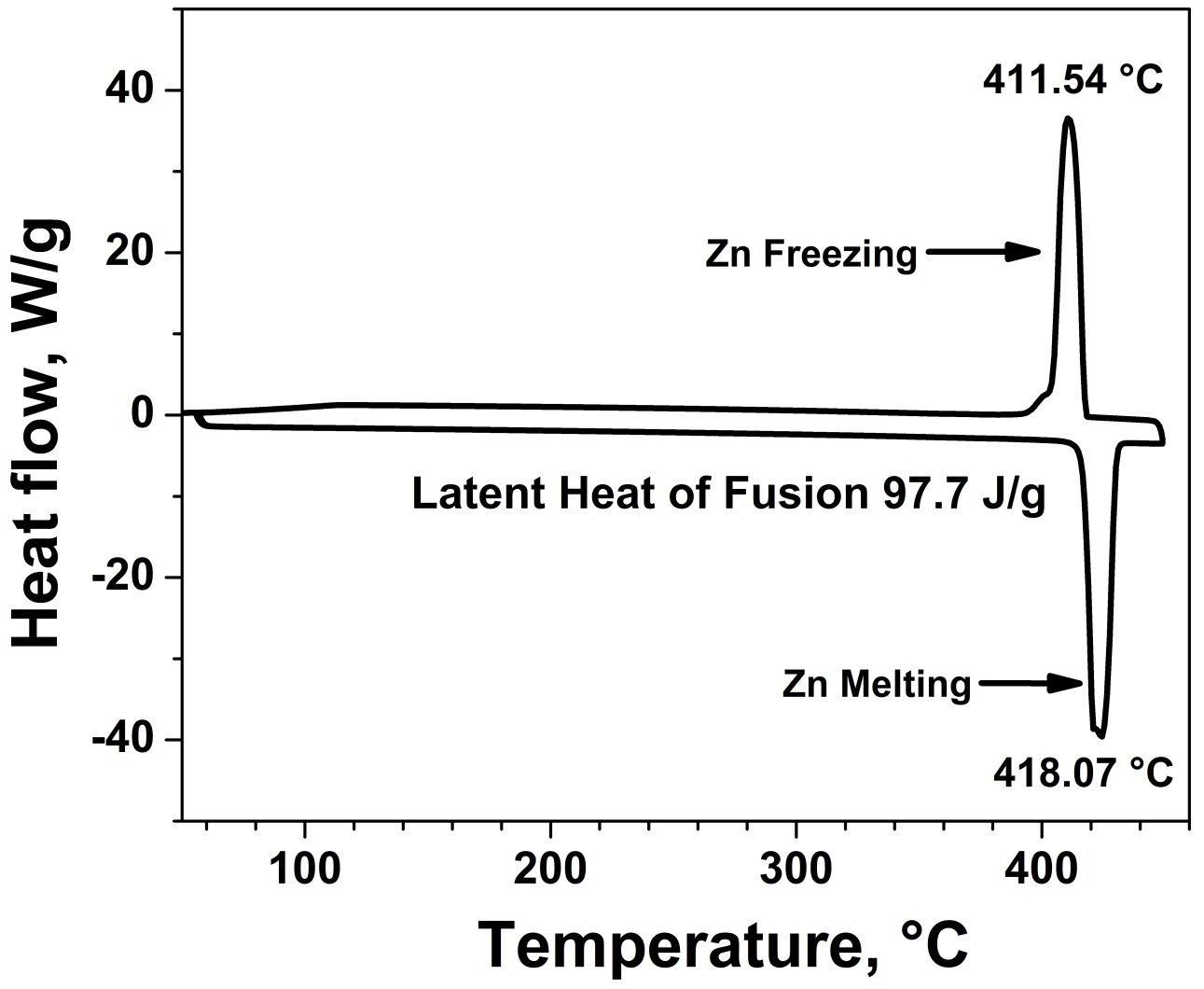

473

474 Figure 3: A typical DSC graph of as-received $\mathrm{Zn}$ particles showing melting and freezing curves. 

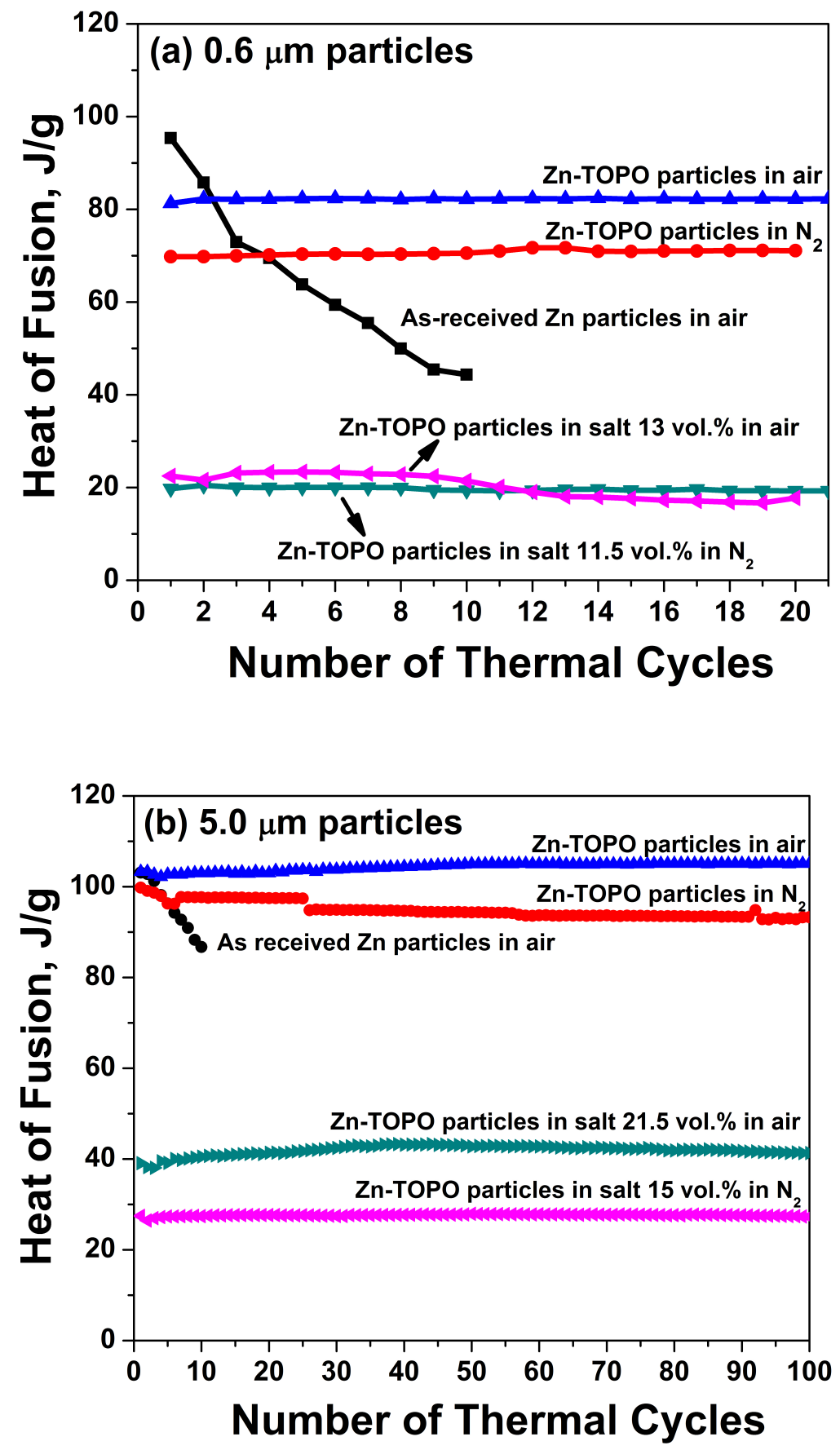

477 Figure 4: Latent heat of fusion (a) for samples with $0.6 \mu \mathrm{m} \mathrm{Zn}$ particles; (b) samples with $5 \mu \mathrm{m}$ 478 Zn particles. 

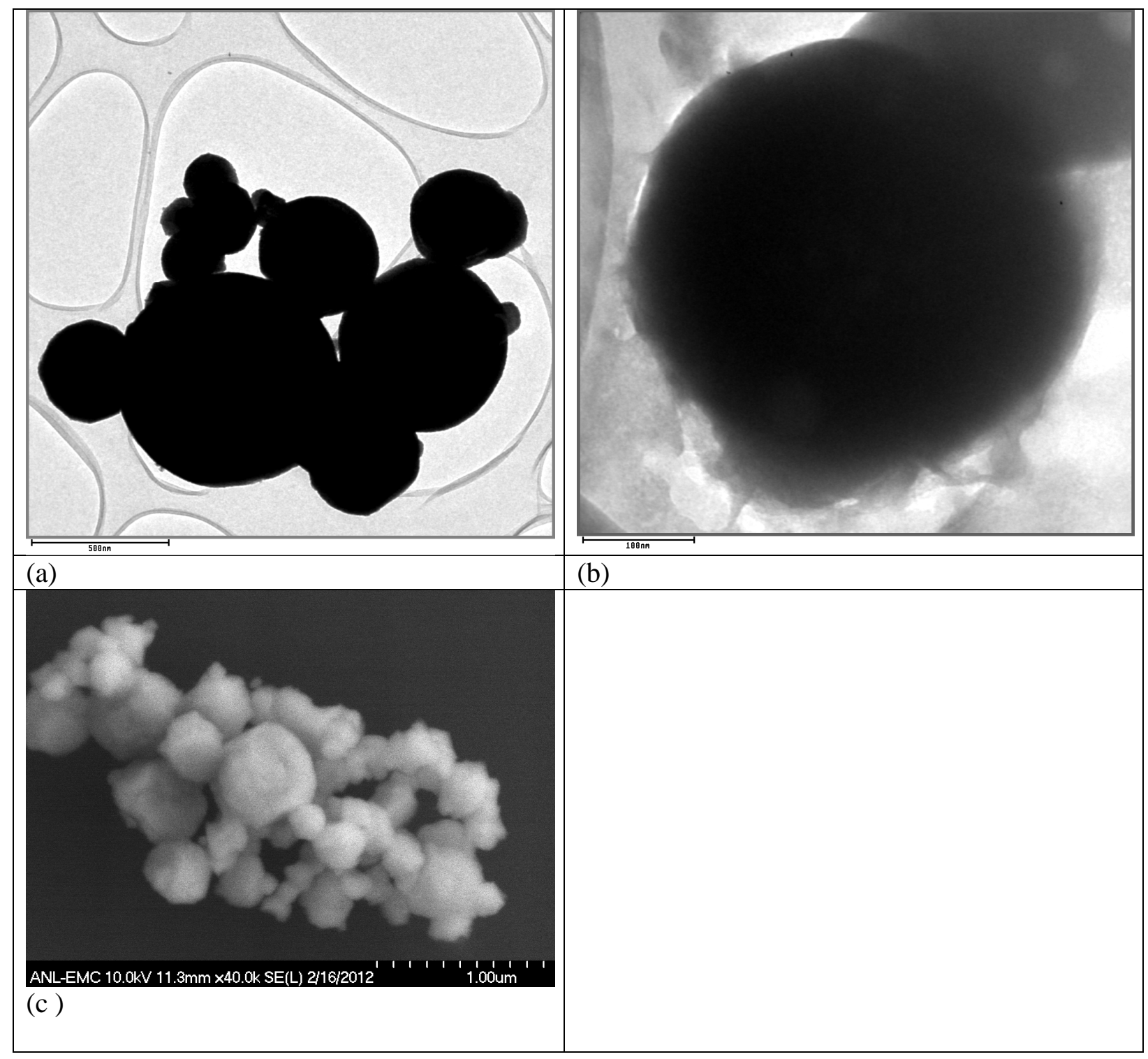

Figure 5: (a) TEM image of TOPO coated $0.6 \mu \mathrm{m} \mathrm{Zn}$ particles before and (b) after 20 thermal 482 cycles of composite and (c) SEM image after 40 thermal cycles of composite (salts were washed 483 off with ethanol). 


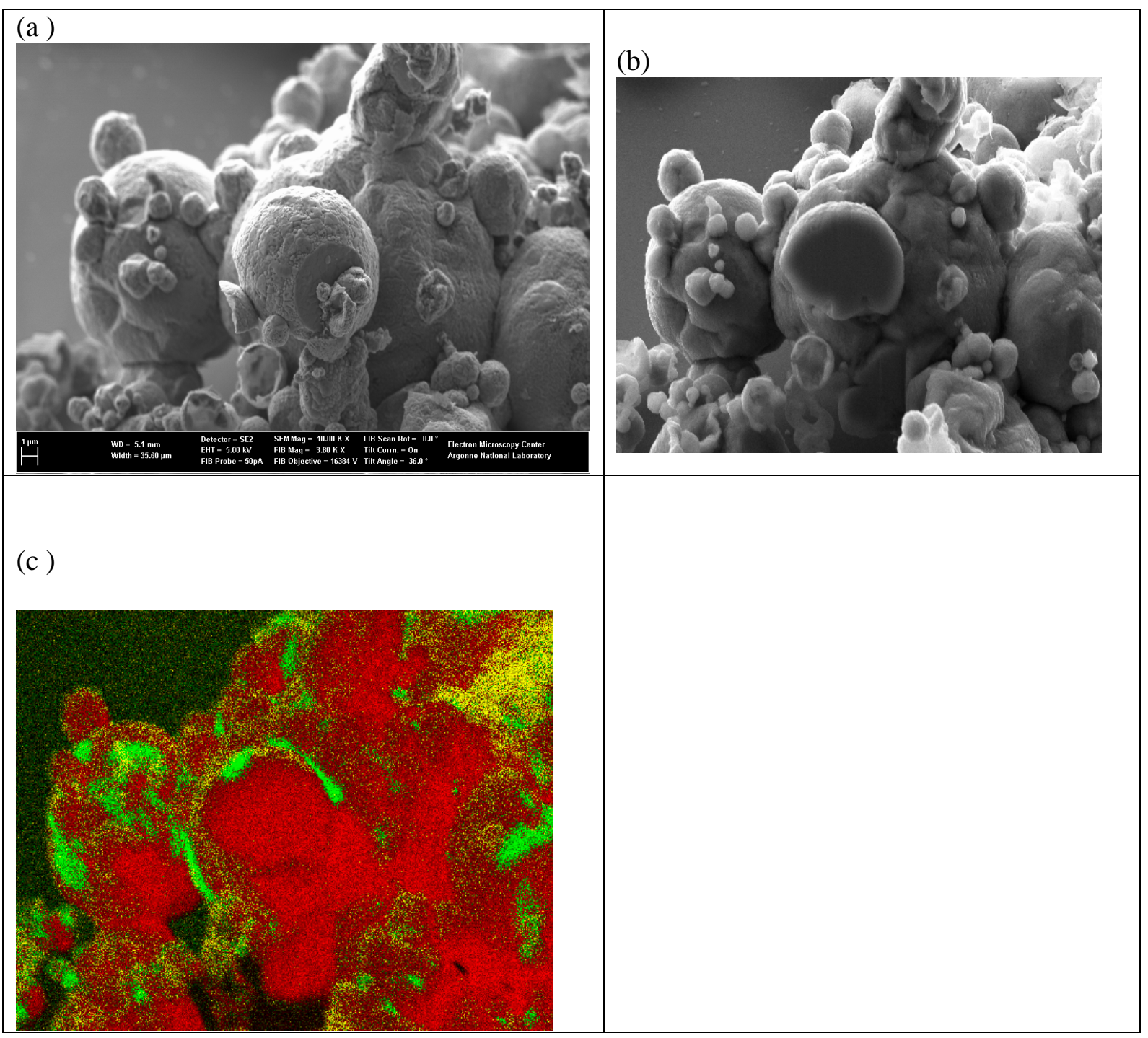

Figure 6: $\mathrm{Zn}$ particles $(5 \mu \mathrm{m})$ exposed to air at $400^{\circ} \mathrm{C}$ show significant surface oxidation. (a ) SEM image showing considerable surface oxidation; (b) FIB-sliced $5 \mu \mathrm{m} \mathrm{Zn}$ particle corresponding elemental mapping (Color codes: Zn-red, O-Yellow). 

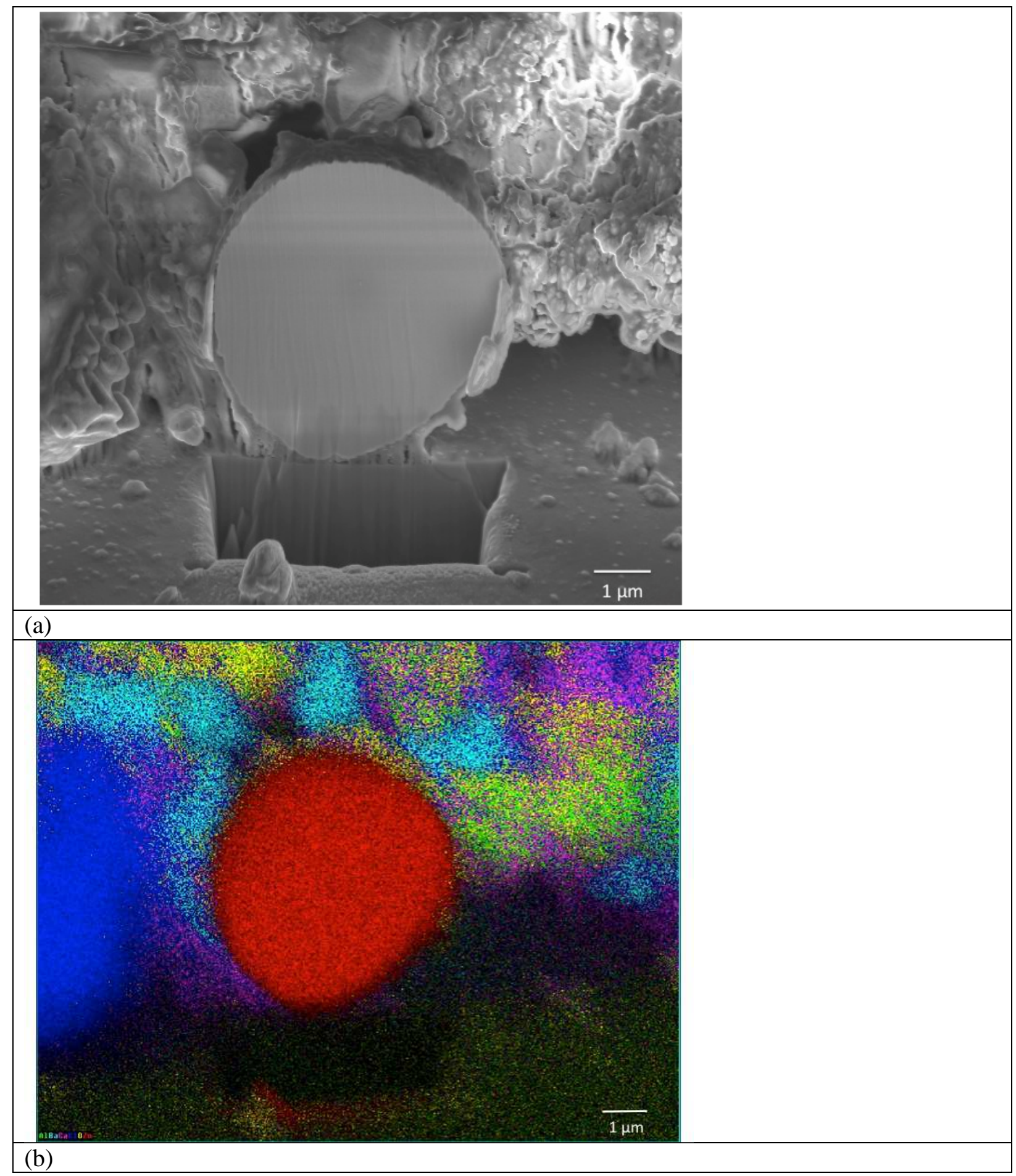

Figure 7: (a) SEM image of composite after 200 thermal cycles in nitrogen atmosphere with 489 FIB-sliced $5 \mu \mathrm{m} \mathrm{Zn} \mathrm{particle} \mathrm{(trench} \mathrm{created} \mathrm{from} \mathrm{FIB} \mathrm{slicing} \mathrm{is} \mathrm{visible),} \mathrm{(b)} \mathrm{corresponding}$ 490 elemental mapping (Color codes: Zn-red, O-yellow, Cl-blue, Ca-purple, Ba-Magenta). 

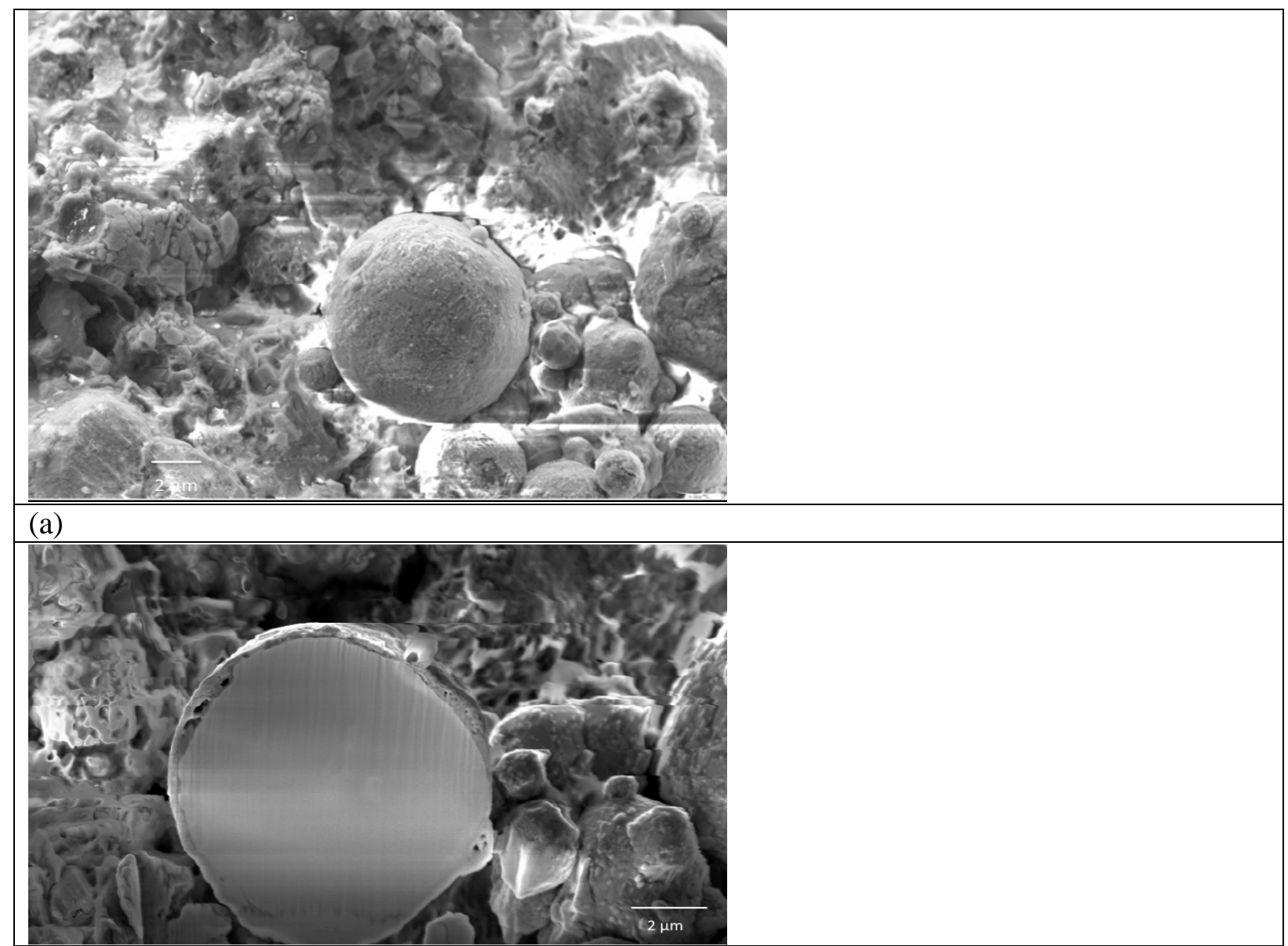

(b)

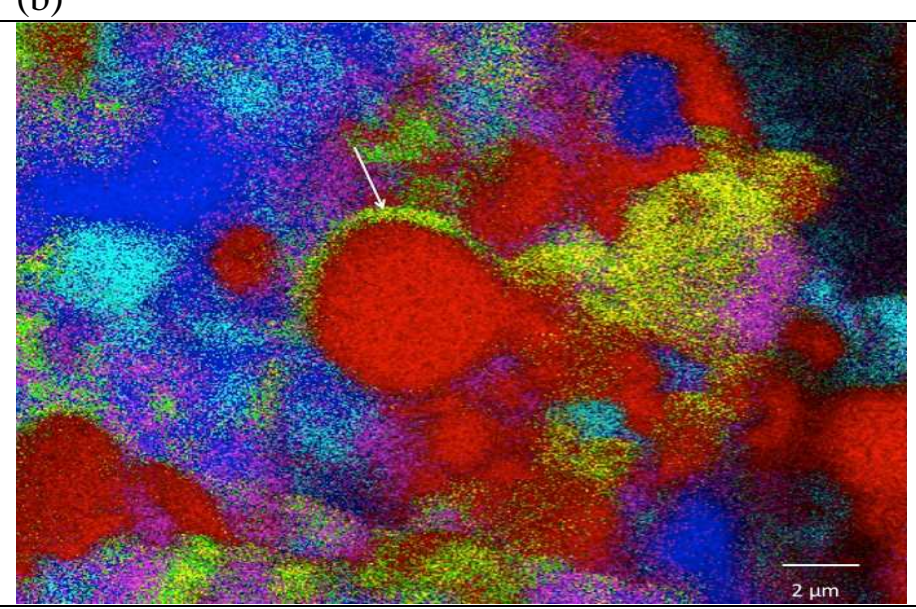

(c) 


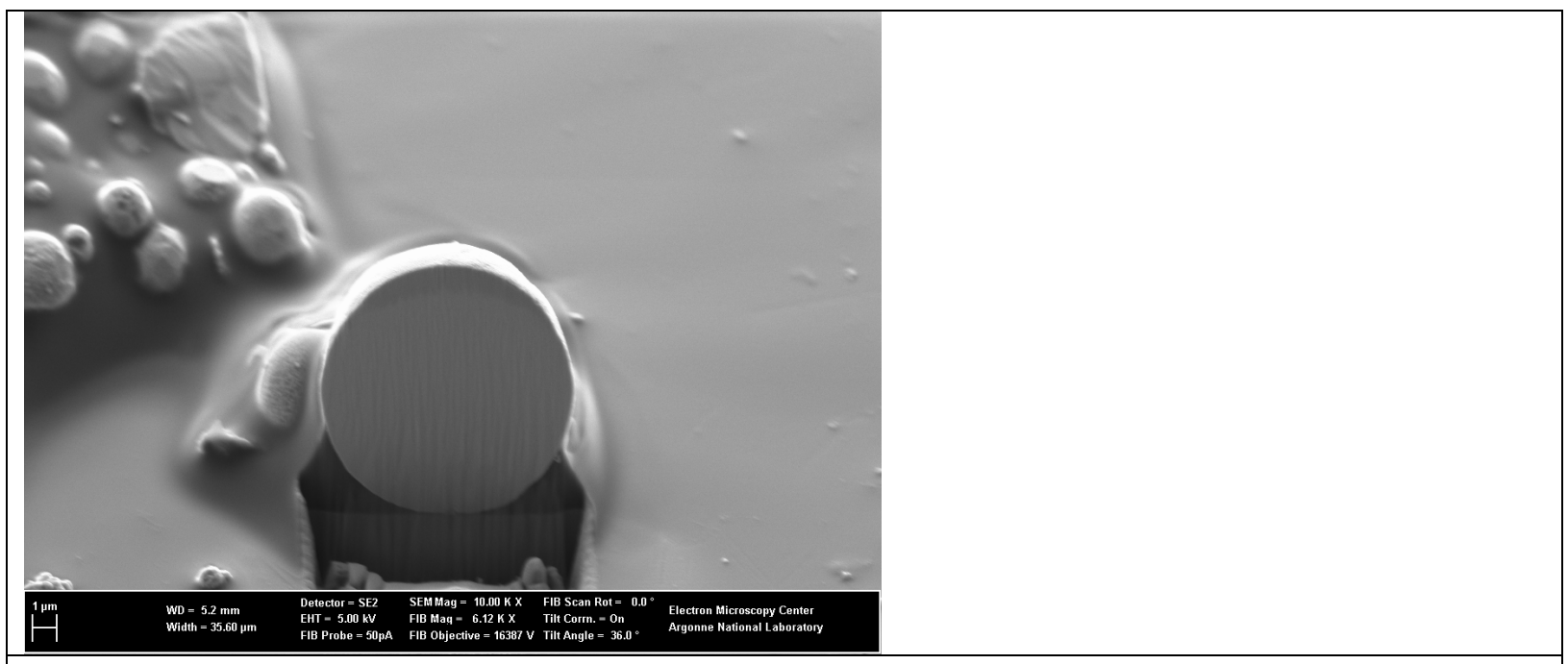

(d)

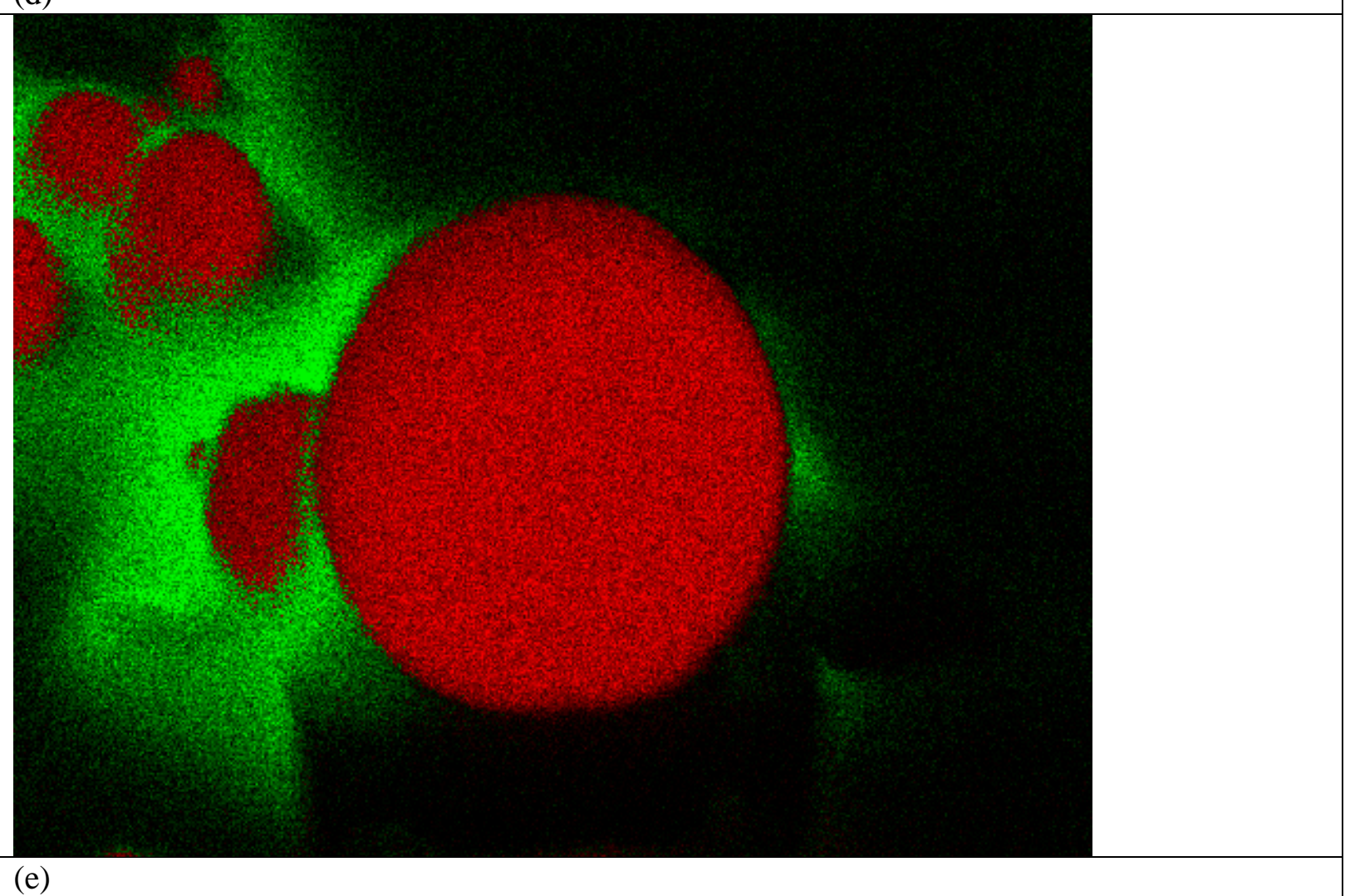

Figure 8: (a) SEM image of the coated Zn particles in eutectic salt after 200 thermal cycles in air 492 atmosphere, (b) SEM image of composite after 200 thermal cycles in air atmosphere with FIB493 sliced $5 \mu \mathrm{m} \mathrm{Zn}$ particles (part of the TOPO coating on the $\mathrm{Zn}$ particle is visible, (c)

494 corresponding elemental mapping, (Color code: Zn-red, O-yellow, Cl-blue, Ca-purple, Ba-

495 Magenta, Al-green). Arrow shows the TOPO coating; (d) FIB-Sliced TOPO coated Zn-

496 nanoparticle without any salts; (e) corresponding elemental mapping showing pure $\mathrm{Zn}$ with no 497 oxygen. 


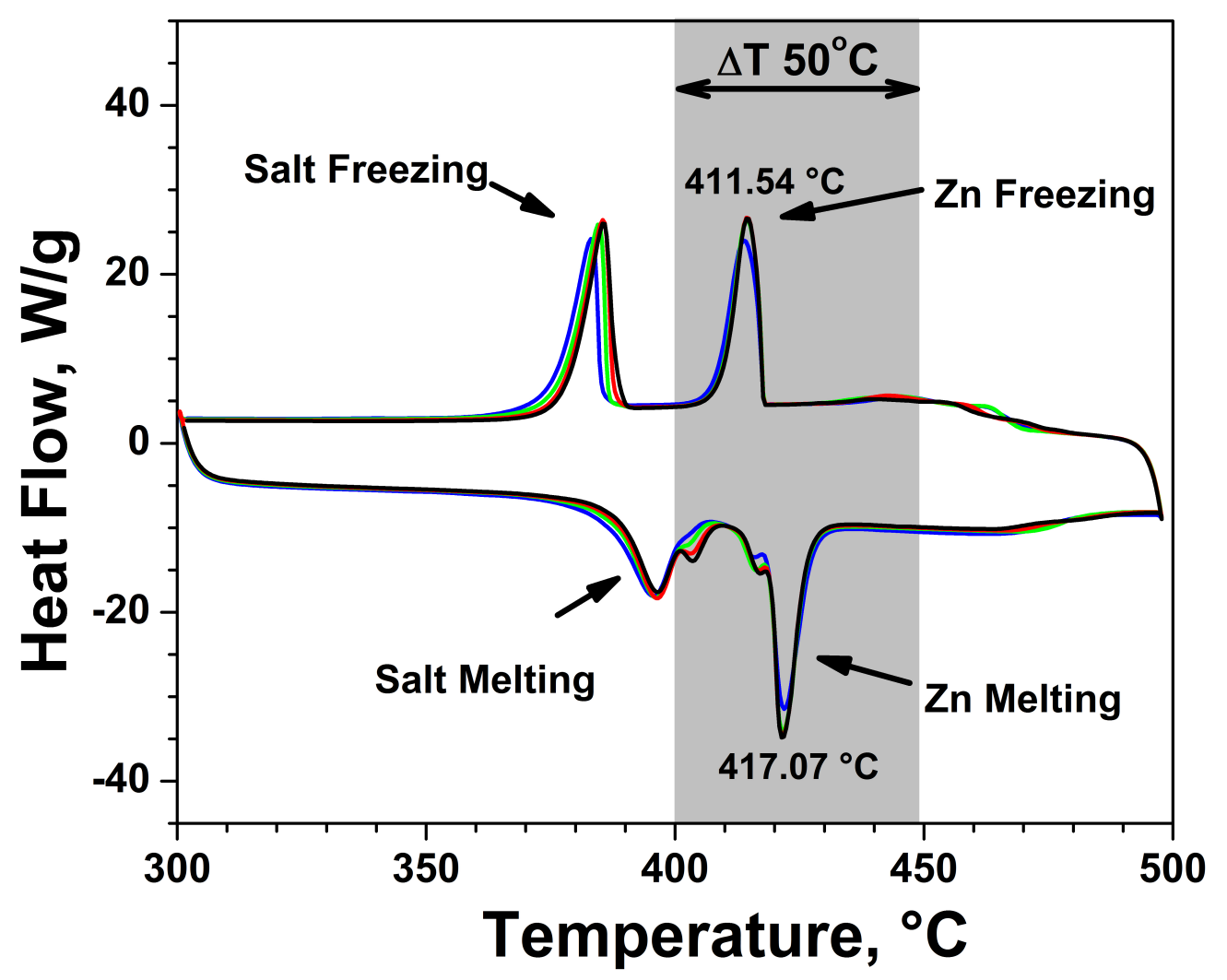

498

Figure 9: DSC graph of TOPO coated Zn particles $(0.6 \mu \mathrm{m})$ at $8.5 \mathrm{vol} . \%$ loading in alkali 500 chloride eutectic salt with repeated heating and cooling cycles between $300^{\circ} \mathrm{C}$ and $500^{\circ} \mathrm{C}$. 501 Melting and freezing of Zn-nanoparticle is highlighted with the shaded background. 


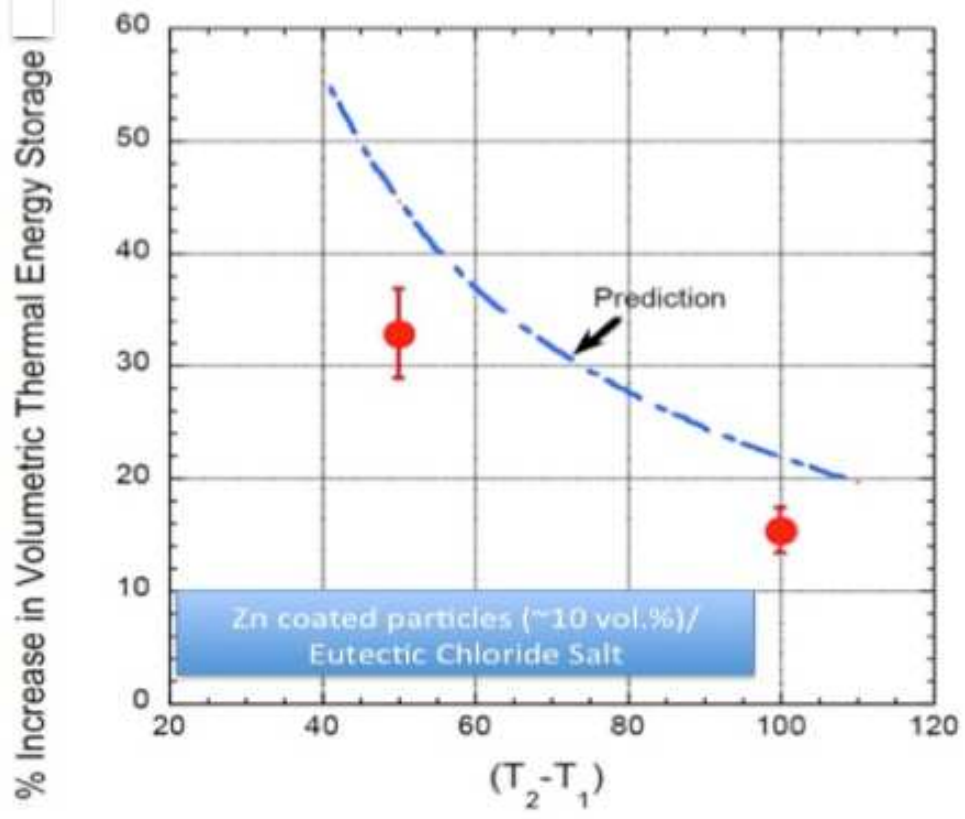

504

505 Figure 10: Average increase in volumetric TES capacity for $10 \mathrm{vol} \%$ loading of EPC-Zn

506 particles $(0.6 \mu \mathrm{m})$ in salt eutectic as a function of the cycling temperature range $(\Delta \mathrm{T})$ for the 507 composite with run to run experimental error bars $(\phi)$. The line curve represents the calculated 508 values. 